\title{
NHLBI Coronary Artery Dissection Type F
}

National Cancer Institute

\section{Source}

National Cancer Institute. NHLBI Coronary Artery Dissection Type F. NCI Thesaurus.

Code C119593.

Non-A-E types with total coronary occlusion and no distal antegrade flow. May represent thrombus. (Adapted from: Coronary artery ang iographic changes after PTCA: Manual of Operations NHLBI PTCA Registry 1985-6:9) 